\title{
Experimental investigation and modeling of nonlinear, adaptive dashpot
}

\author{
K. Mnich $\cdot$ M. Lazarek $\cdot$ P. Brzeski $・$ P. Perlikowski $\mathbb{D}$
}

Received: 21 March 2020/Accepted: 18 June 2020/Published online: 6 July 2020

(C) The Author(s) 2020

\begin{abstract}
In this paper we determine the characteristics of mechanical components of dynamical systems using a specially designed laboratory rig. We present the details of the experiments performed on the prototype device and provide technical documentation of its crucial elements. We validate the accuracy of measurements using a spring and comparing results with manufacturer data. Then, we examine nonlinear dashpot with variable damping coefficient obtained through the usage of the throttling valve. We perform several tests presenting its force characteristics as a function of velocity and damping coefficient. We derive the mathematical model of the dashpot basing on the experimental data. Finally, we perform transient test in which we change the damping coefficient during operation of the dashpot. The comparison of obtained results with the model gives good accordance.
\end{abstract}

Mnich and Lazarek have contributed eually to this work.

Electronic supplementary material The online version of this article (https://doi.org/10.1007/s11012-020-01196-0) contains supplementary material, which is available to authorized users.

K. Mnich · M. Lazarek · P. Brzeski · P. Perlikowski ( $₫)$ Division of Dynamics, Lodz University of Technology, Stefanowskiego 1/15, 90-924 Lodz, Poland

e-mail: przemyslaw.perlikowski@p.lodz.pl
Keywords Nonlinear dashpot - Semi-active dashpot $\cdot$ Experimental test rig $\cdot$ Experimental model

\section{Introduction}

The engineering constructions are often subjected to unwanted vibrations that in some cases can be mitigated by means of a viscous damper. It is a device that dissipates the excess of the kinetic energy thanks to the viscosity of the fluid moving inside. As the layers of a fluid move against each other, the kinetic energy is changed into heat and dissipated to the environment [1,2]. Dampers of this kind are widely used in civil engineering [3, 4], aerospace [5-7] and automobile $[8,9]$ industries. In the last listed field, a viscous damper is often called dashpot and is the most frequently used as a part of the vehicle suspension. We can distinguish the linear and rotary dashpots, with respect to the manner in which they perform motion. Nevertheless, direction of motion should not be confused with velocity-force characteristic which can be either linear or non-linear in both types of dashpot. The simplest devices are designed to be linear what corresponds with the assumption of the purely linear characteristics hence the force generated in the dashpot is proportional to relative velocity of device terminals. The more precise model takes into account that the force is proportional to the relative velocity 
raised to required power, that depends on a particular dashpot construction [3, 10-12]. The damping force depends on the geometry of the dashpot and of the fluid used. Both factors are optimized to suite the working conditions of the device such as the frequency and the power of damped oscillations. In some applications the working conditions of the damped system change over time and there is a need to vary the characteristics of the damper. Such a device is called the semi-active damper and it is used in many industrial applications. For example, one could expect a car suspension to behave differently for varying road conditions [13-15]. A controller connected to a system of sensors monitors the working conditions and adjusts the damping properties to the current needs. Dampers with variable damping coefficients are also widely used in mechanical and structural systems for seismic and wind storms protection of high buildings [16-18], control of vibrations of wind turbines [19-22], stabilizing washing machines [23], chatter suppression [24, 25], high impulsive loads [26-28], stabilization of flexible structures [29] and many more. One can also find applications in medicine where the damper is a part of prosthesis [30, 31]. As the damping depends on the geometry of the dashpot and on the fluid viscosity, the adjustment can be done in two ways. First of the popular solutions relies on the properties of the magnetorheological fluid [32]. Its viscosity can be controlled by changing the magnetic field around $[7,33]$, typically by changing the current in electromagnets. The other solution is based on the change of geometry of the flow. An adjustable throttling valve locally changes the cross section of the flow $[34,35]$ restraining or relaxing the motion of the fluid.

In this article a semi-active dashpot with a throttling valve is experimentally analyzed. We design the dedicated rig that enables to measure the acting force of damper at different relative velocities of its nodes and at different positions of the throttling valve. We show the mathematical model of investigated device that match perfectly to experimental data. Presented results are a part of the on going work on the properties of the tuned mass damper with the adjustable dashpot [36-38].

The paper is organized as follows. In Sect. 2 we describe the test stand. Section 3 is devoted to validation of correctness of all components of the experimental rig. We describe measurements of the stiffness of the spring and extensive experimental tests of the damper. In Sect. 4 we create a mathematical model of the dashpot basing on previously obtained experimental results. Then, in Sect. 5 we validate the model experimentally simulating the continuous changes of damping coefficient. The results are concluded in the last section.

\section{Description of the test stand}

The laboratory rig (Fig. 1) was specifically designed to examine elementary components of dynamical systems like springs, dashpots and inerters. It has main dimensions of $1020 \times 110 \times 300(\mathrm{~mm})$. The test stand is placed in the laboratory of the Division of Dynamics at the Lodz University of Technology in Poland. The description of the rig is made basing on a setup prepared to examine a dashpot. The base is composed of a steel, H shaped beam (HEB 100) (part No. 1) with six leveling feet (part No. 2) fastened to the profile through the threaded holes and countered from the bottom by the nuts. The base profile provides stiffness needed in the experiments, while the feet allow a proper leveling mainly due to the uneven surface of the laboratory floor. The profile is fixed to the ground through the bolts blocked in the T-shaped grooves in the floor. On the top of the profile there is a $15(\mathrm{~mm})$ thick milled plate (part No. 3) made of EN AW 7021 aluminum alloy fixed to the beam with the bolts. The plate ensures a high flatness and creates the base for the key components of the system. The main part of the rig is a linear actuator Unimotion PNCE 50 (part No. 4). It is placed at the end of the plate and is mounted through the intermediate platforms (part No. 5) designed to gain vertical space needed to perform the experiments on the larger objects. The actuator can withstand the velocity up to $1\left(\frac{\mathrm{m}}{\mathrm{s}}\right)$, the acceleration up to $20\left(\frac{\mathrm{m}}{\mathrm{s}^{2}}\right)$ and the input torque below $14(\mathrm{~N} \mathrm{~m})$. According to a convention chosen in this work, the positive sign of the velocity value indicates, that the actuator is pulling. The maximal stroke of the piston rod is equal to $300(\mathrm{~mm})$. The ball screw used in the actuator provides $20(\mathrm{~mm})$ of stroke per one revolution of the drive. The actuator is driven by the Mitsubishi servomotor HGJR203b (part No. 6), with the nominal power of $3(\mathrm{~kW})$ and the nominal torque of $17(\mathrm{Nm})$. The servomotor is equipped with the absolute encoder with the resolution of 


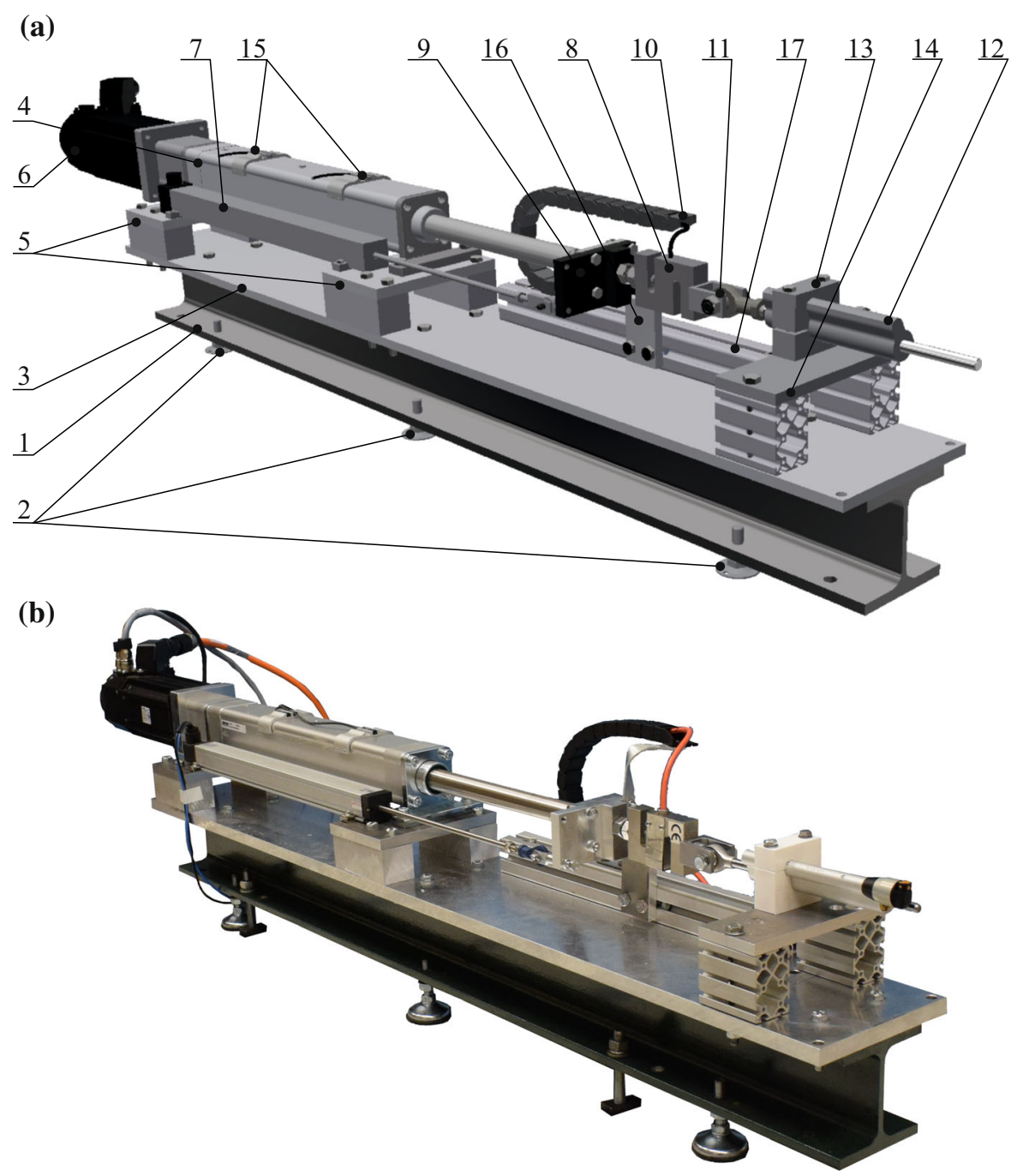

Fig. 1 Design of the laboratory rig a and its realization (b). Parts presented in Figure: 1-Steel beam HEB100, 2-Leveling feet, 3-Aluminum plate, 4-Electric actuator Unimotion PNCE50, 5-Intermediate platforms, 6-Mitsubishi servomotor HGJR203b, 7-Linear potentiometer KTC-375, 8-Load

4,000,000 (PPR). The encoder is a part of the driver's closed loop system but is also used as the main position sensor. An additional linear position sensor (part No. 7) is mounted in parallel to the actuator. It is a linear potentiometer KTC-375 whose resistance is $4(\mathrm{k} \Omega)$. Measurement of the force generated by the examined devices is possible due to a force sensor (part No. 8) installed at the end of the piston rod. The universal mounting bracket (part No. 9) between the sensor and the piston rod is designed to ensure a possibility of using different models and types of cell m620, 9-Universal mounting bracket, 10-Cable guiding Igus R07, 11-Spherical joint and a fork, 12-Dashpot Ohlins SD 043, 13-Dashpot bracket, 14-Dashpot platform, 15Proximity sensors SMT 65, 16-Mechanical brake, 17-Linear guiding for the mechanical brake

sensors. The same mounting bracket is used to fasten the piston rod of the potentiometer. For the experiment we use an s-type load cell m620 nominally measuring the forces up to $500(\mathrm{~N})$, that can be safely overloaded to $1000(\mathrm{~N})$. In order to protect the cable of the sensor, we put it in a plastic cable chain Igus R07 (part No. 10). The second end of the force sensor is connected to the dashpot through a combination of spherical and cylindrical joints (part No. 11). This type of connection reduces the minimal assembly misalignment. The connection can also be easily used to mount various 
types of devices such as springs, dashpots and inerters. In this particular experiment we examine the semiactive dashpot Ohlins SD 043 (part No. 12). The stroke of the dashpot is equal to $60(\mathrm{~mm})$. It has an adjustable damping coefficient, regulated by a built in stepper motor. The motor drives a throttling valve, which changes the cross section of the channel through which the damping liquid is flowing. The dashpot is mounted in a specially designed bracket (part No. 13) fixed to a platform similar to the one put under the actuator (part No. 14).

The test rig is controlled through a PC connected to the driver of the servomotor via the USB interface. By means of the dedicated software-Mitsubishi MR Configurator 2 - the position, the velocity and the acceleration of the motor in time can be precisely adjusted. The motor we use has a power reserve so in case of the software failure it could easily destroy the other components of the rig, therefore we applied the following preventive measures. Firstly, the torque of the motor is software-limited to $20 \%$ of the nominal value resulting in $3.4(\mathrm{~N} \mathrm{~m})$, which corresponds to $1000(\mathrm{~N})$ of the axial thrust. This is the maximal load that the force sensor can stand. Secondly, close to the piston rod stroke limits, there are two magnetic field proximity sensors SMT 65 (part No. 14), which act as safety switches. When the piston rod exceeds the limits imposed by the sensors they stop the servomotor. Finally, we placed an adjustable mechanical brake (part No. 15), on which the piston rod of the actuator will stop if both the software and the switches fail. A position of the brake can be adjusted along the aluminum profile Item $840 \times 40$ (part No. 16) according to the accessible stroke of the object under investigation. Also for the safety reasons we limit the acceleration of the drive.

The damping of the dashpot is also regulated from the PC, through an own software written in Ansi C. The software is executed by an Arduino UNO board used as a signal generator. Then the motor is driven by a driver equipped with double h-bridge L298N. The interface of the program lets us to increase and decrease the damping within the limits defined from 0 to 130 (-) with a resolution of 1 (-) unit.

Data acquisition is done through a National Instruments card NI USB-6259. It has 16 analog differential input channels with the resolution of 16 bits each. It is capable to sample with the rate of $1\left(\frac{\mathrm{MS}}{\mathrm{s}}\right)$ and accepts the inputs from -10 to $10(\mathrm{~V})$.

\section{Experimental results}

In the first part of this section, we show experimental validation of the test rig. While in the second part, we analyze semi-active damper with variable damping coefficient presenting several velocity-force characteristics for different values of damping coefficient.

\subsection{Validation of the test rig}

To validate the correct assembly and operation of the test rig we perform series of tests. In all of them the device has been equipped with three 3-axial accelerometers (one mounted on the end of the piston of the linear actuator, one on the dashpot and one on the rig) to measure oscillations of the system during operation. Based on accelerograms we are able to minimize vibrations in directions perpendicular to the axis of motion by slight corrections of the assembly of all elements in measurement chain and ensure their coaxiality. The next stage of test has been focused on comparison between assumed velocity and acceleration profiles assumed in control software and real motion of the device.

In the very last test we measure the characteristic of a linear helical extension spring of a known stiffness. According to the supplier documentation the spring used in the experiment has the stiffness of $3.29\left(\frac{\mathrm{N}}{\mathrm{mm}}\right)$, 26.5 coils, wire diameter equal to $1.8(\mathrm{~mm})$, external diameter of $12.5(\mathrm{~mm})$, free length of $63(\mathrm{~mm})$ and is ended by the German hooks (Vanel U.125.180.0630.A). The spring is preloaded with the force of $10(\mathrm{~N})$ in order to overcome the initial tension of the spring and to remove the play present in the system. Next, it is elongated by $40(\mathrm{~mm})$ and obtain force - displacement relation. The stiffness of the spring is derived from the linear fit obtained by the method of least squares. The measurement has been repeated ten times and the coefficient of determination $R^{2}$ of each fit has been above 0.99 . The mean stiffness coefficient is equal to $3.29\left(\frac{\mathrm{N}}{\mathrm{mm}}\right)$. To be sure that there is no relation between the measured force and the velocity of motion, the spring has been also elongated 
at different velocities of the actuator and indeed no relation has been found.

\subsection{Experiment on the dashpot}

The characteristics of the dashpot have been investigated with respect to the velocity of the piston rod and damping coefficient of the dashpot. As aforementioned in Sect. 2 the damping coefficient of the semiactive damper is controlled by a built-in stepper motor. Let us introduce $\gamma$ parameter describing position of the stepper motor that can vary from 0 to $130(-)$. The force generated by the dashpot has been measured for the velocities from 0.033 to $0.30\left(\frac{\mathrm{m}}{\mathrm{s}}\right)$ with the step of $0.033\left(\frac{\mathrm{m}}{\mathrm{s}}\right)$ The step value comes from the main servomotor whose velocity is regulated in revolutions per minute, hence change of 100 (rpm) of servomotor results in a change of $0.033\left(\frac{\mathrm{m}}{\mathrm{s}}\right)$ in the linear motion of the actuator. The control parameter is adjusted at each velocity from 0 to $130(-)$ with the step of $10(-)$ units (it results in change of damping coefficient of the dashpot). Figure 2 shows a general trapezoidal velocity profile used in the experiment. The dashpot is accelerated in the interval from $t_{0}$ to $t_{1}$ and decelerated from $t_{2}$ to $t_{3}$. The velocity is constant from $t_{1}$ to $t_{2}$. The time intervals are adjusted in such a way, that the distance traveled by the piston rod of the dashpot is always the same and equal to $5(\mathrm{~mm})$ during acceleration, $40(\mathrm{~mm})$ in the constant velocity phase and $5(\mathrm{~mm})$ during deceleration, which sums up to $50(\mathrm{~mm})$. The maximal stroke of the dashpot is equal to $60(\mathrm{~mm})$, but $5(\mathrm{~mm})$ of the safety margin were left from the both ends of the piston rod. The maximum investigated velocity is defined by the maximum allowable acceleration of the actuator, equal to $20\left(\frac{\mathrm{m}}{\mathrm{s}^{2}}\right)$.
An experimental realization of the general profile from Fig. 2 is shown in Fig. $3 \mathrm{a}$ for the maximum velocity equal to $0.066\left(\frac{\mathrm{m}}{\mathrm{s}}\right)$. Figure $3 \mathrm{~b}$ shows the force measured at this velocity for the control parameter set to $\gamma=100(-)$. It is worth to notice, that the force in Fig. 3b starts increasing before the motion starts. This effect comes from the dry friction present in the dashpot. We assume, that the velocity is constant when its value is within the range $v \pm 1 \% v$, where $v$ is the expected value of constant velocity. Then, the force $F$ is measured in the whole range of constant velocity and as a result we take the mean value of force. Such a value corresponds to one measurement point presented in Fig. 4, which shows a summary of all measurements taken in the experiment. The measurement points are marked as dots while the lines are the linear interpolations between the points which are taken for the same value of damping coefficient. Figure 4 contains 14 curves representing the velocity-force characteristics of the dashpot measured for 14 different damping coefficient values controlled by control parameter $\gamma$. Effect from the dry friction present in the dashpot is also reflected in this figure, as the curves do not start at $0(\mathrm{~N})$. It is clear that the dashpot has a non-linear characteristic with visible hardening effect according to increase of velocity of piston rod. Moreover, increase of parameter $\gamma$ is causing an increase in non-linearity of the dashpot response, i.e., the larger $\gamma$, the stepper increase of damping force. The movie showing sample test of the semi-active dashpot is attached in Supplementary Content.

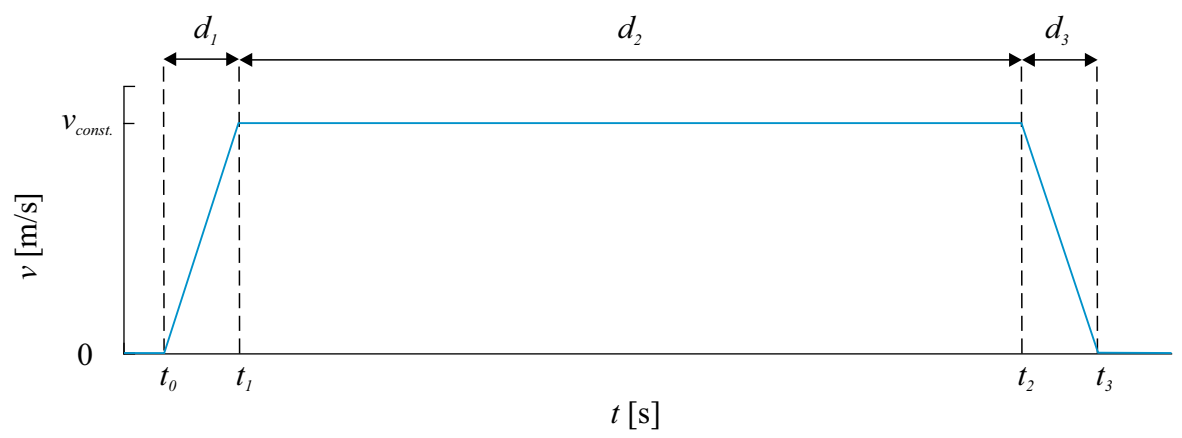

Fig. 2 Velocity profile used in the experiment. The actuator is accelerated from $t_{0}$ to $t_{1}$ on the path of $d_{1}=5$ ( $\left.\mathrm{mm}\right)$. From $t_{1}$ to $t_{2}$ the velocity is constant on the distance of $d_{2}=40(\mathrm{~mm})$ and from $t_{2}$ to $t_{3}$ the actuator decelerates on the path $d_{3}=5(\mathrm{~mm})$ 


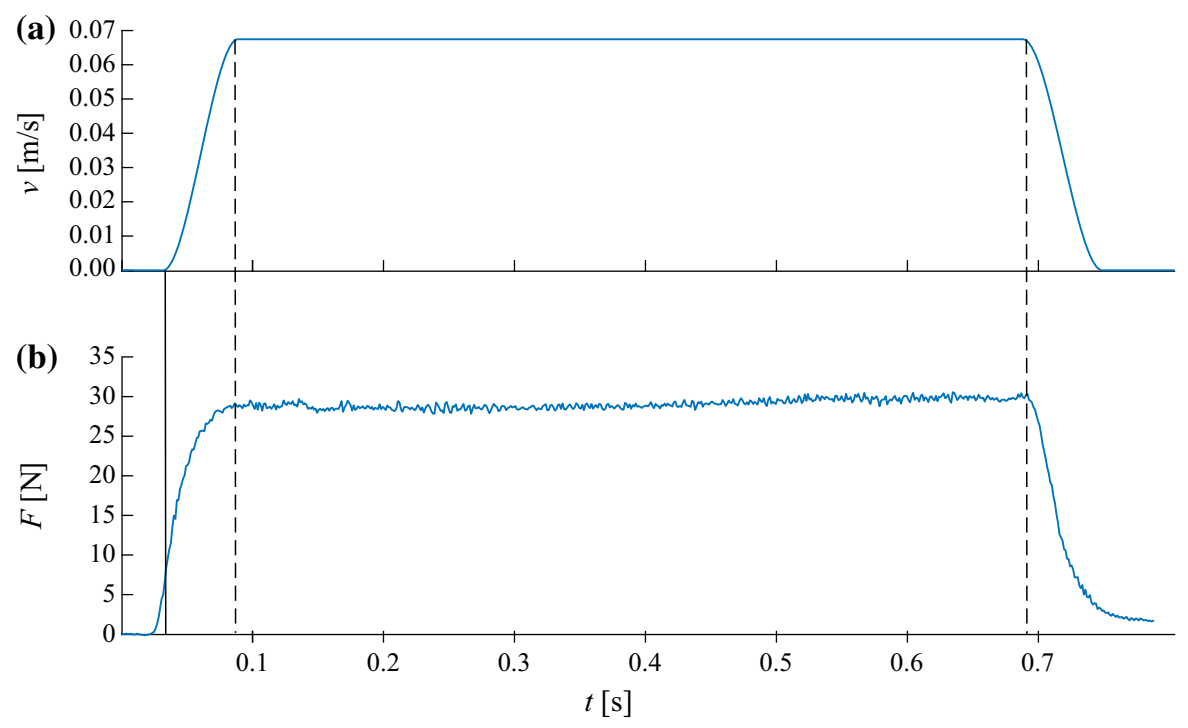

Fig. 3 Time series of the velocity of the piston rod measured with the main servomotor encoder (a) and the force pushing the piston rod (b) for the control parameter adjusted to $\gamma=100(-)$. The vertical dashed lines indicate the time interval in which the

\section{Model of the dashpot}

The measurements performed for the investigated dashpot are used to create its mathematical model. The force $F$ generated by the device has been measured with respect to two variables, absolute velocity $v$ and control parameter $\gamma$. Hence, we are able to create a two parameter surface given by a function $F=f(v, \gamma)$. We fit a polynomial surface to the data points by the RMS method. The order of polynomial is equal to three and five in $v$ and $\gamma$ directions respectively. It is the lowest order of polynomial, that reflected well all the changes of the measured force and it results in very good fitting given by $R^{2}=0.999$. The obtained formula is as follows: piston rod moves with the velocity $v=0.066\left(\frac{\mathrm{m}}{\mathrm{s}}\right) \pm 1 \%$. The solid vertical line shows the beginning of the motion. The force is not equal to zero at this point because of the dry friction

where $\quad p_{00}=6.902(\mathrm{~N}), \quad p_{10}=170\left(\mathrm{~N} \frac{\mathrm{s}}{\mathrm{m}}\right)$, $p_{01}=0.6723(\mathrm{~N}), \quad p_{20}=-283.3\left(\mathrm{~N} \frac{\mathrm{s}^{2}}{\mathrm{~m}^{2}}\right)$, $p_{11}=2.66\left(\mathrm{~N} \frac{\mathrm{s}}{\mathrm{m}}\right), \quad p_{02}=-0.0493675(\mathrm{~N}), \quad p_{30}=$ $2226\left(\mathrm{~N} \frac{\mathrm{s}^{3}}{\mathrm{~m}^{3}}\right), \quad p_{21}=2.632\left(\mathrm{~N} \frac{\mathrm{s}^{2}}{\mathrm{~m}^{2}}\right)$, $p_{12}=0.01399\left(\mathrm{~N} \frac{\mathrm{s}}{\mathrm{m}}\right), \quad p_{03}=0.001195(\mathrm{~N})$, $p_{31}=-8.396\left(\mathrm{~N} \frac{\mathrm{s}^{3}}{\mathrm{~m}^{3}}\right), \quad p_{22}=0.3708\left(\mathrm{~N} \frac{\mathrm{s}^{2}}{\mathrm{~m}^{2}}\right)$, $p_{13}=-0.001915\left(\mathrm{~N} \frac{\mathrm{s}}{\mathrm{m}}\right), \quad p_{04}=-1.114 \times 10^{-5}(\mathrm{~N})$, $p_{32}=-0.332\left(\mathrm{~N} \frac{\mathrm{s}^{3}}{\mathrm{~m}^{3}}\right), \quad p_{23}=-0.000362\left(\mathrm{~N} \frac{\mathrm{s}^{2}}{\mathrm{~m}^{2}}\right)$, $p_{14}=1.605 \times 10^{-5}\left(\mathrm{~N} \frac{\mathrm{s}}{\mathrm{m}}\right), \quad p_{05}=3.524 \times 10^{-8}(\mathrm{~N})$. We plot the surface based on the introduced equation and we show it in Fig. 5. To present the changes in the

$$
\begin{gathered}
F=f(v, \gamma)=p_{00}+p_{10} \cdot v+p_{01} \cdot \gamma+p_{20} \cdot v^{2}+p_{11} \cdot v \cdot \gamma+p_{02} \cdot \gamma^{2}+p_{30} \cdot v^{3}+p_{21} \cdot v^{2} \cdot \gamma \\
+p_{12} \cdot v \cdot \gamma^{2}+p_{03} \cdot \gamma^{3}+p_{31} \cdot v^{3} \cdot \gamma+p_{22} \cdot v^{2} \cdot \gamma^{2}+p_{13} \cdot v \cdot \gamma^{3}+p_{04} \cdot \gamma^{4} \\
+p_{32} \cdot v^{3} \cdot \gamma^{2}+p_{23} \cdot v^{2} \cdot \gamma^{3}+p_{14} \cdot v \cdot \gamma^{4}+p_{05} \cdot \gamma^{5}
\end{gathered}
$$




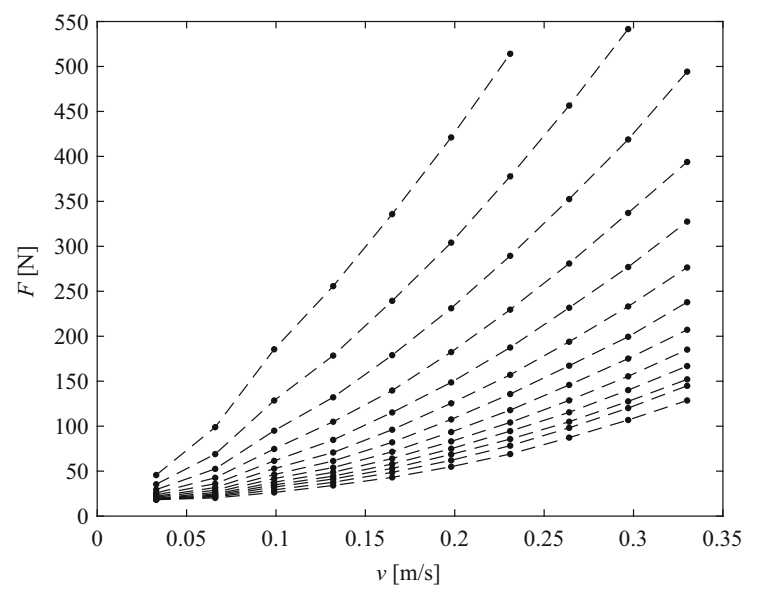

Fig. 4 Velocity-force characteristics of the dashpot determined for the control parameter $\gamma$ varied from $0(-)$ to $130(-)$ with step equal to $10(-)$ units. Dots are measured points while lines are linear interpolation between points taken for the same value of $\gamma$. The characteristic for the lowest value of $\gamma$ corresponds to the lowest line and consecutive lines correspond to increasing values of $\gamma$ up to maximum value

force with increase of velocity and control parameter $\gamma$ we use a color scale for surface, additionally by blue bullets we mark the experimentally measured values of force.

In order to have a better view on the response of the dashpot on changes of two control parameters, we plot two projections of the surface in Fig. 6. In the first projection presented in Fig. 6a we plot 14 forcevelocity characteristics for $\gamma \in\langle 0,130\rangle(-)$ with

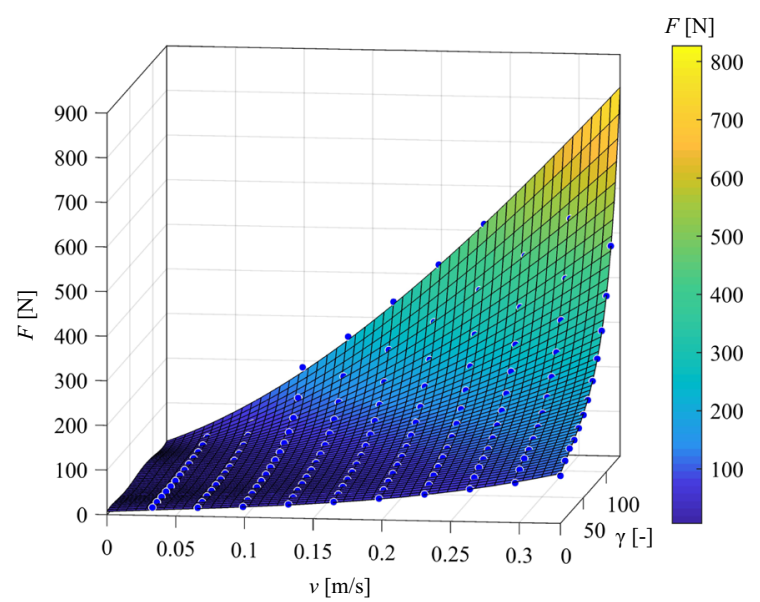

Fig. 5 Force $F$ presented as a two dimensional surface $f(v, \gamma)$. The polynomial surface is fit to the measurement points (blue bullets) by the RMS method with $R^{2}=0.999$. Change of the surface color corresponds to force value
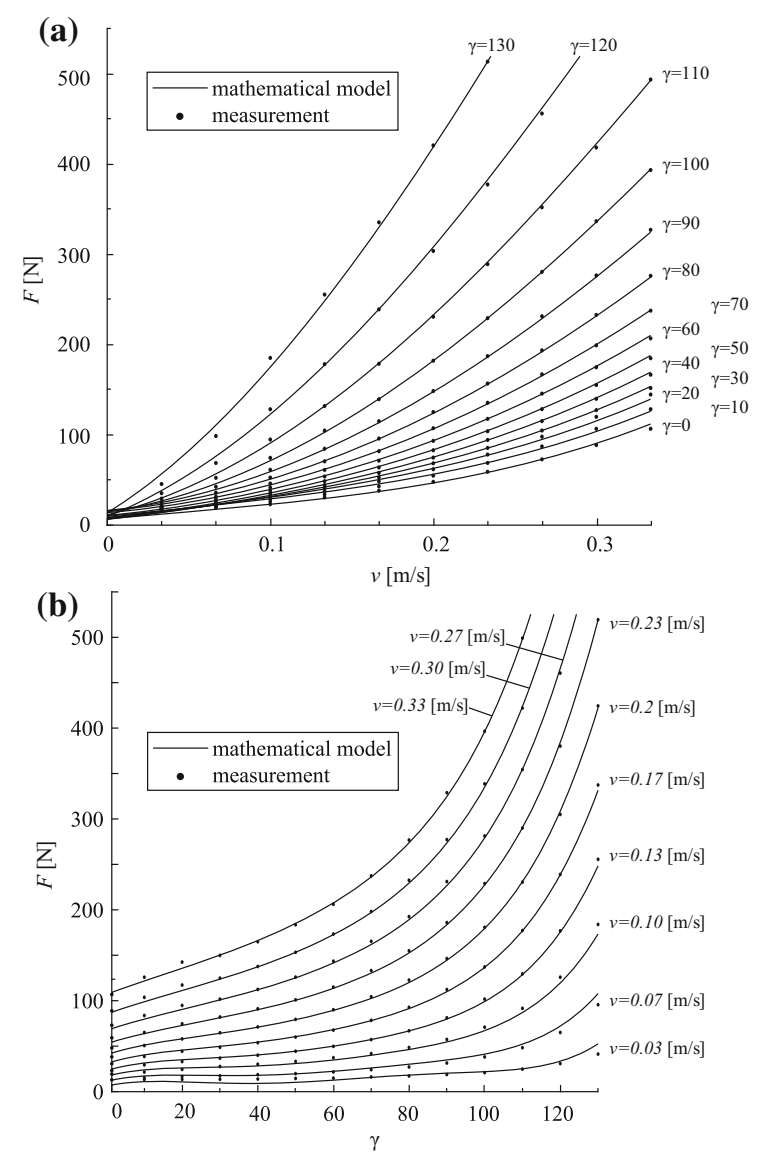

Fig. 6 The cross sections of the surface presented in Fig. 5. a Force at 14 different damping setups $\gamma$ from 0 to 130 as the functions of velocity $v$. b Force at 10 different velocities $v$ from $0.03\left(\frac{\mathrm{m}}{\mathrm{s}}\right)$ to $0.33(\underline{\mathrm{ms}})$ as the functions of control parameter $\gamma$. Both graphs contain the same set of measurement points marked with dots

$10(-)$ units step. The mathematical model fits well with measured data (marked by dots). Results visible in this plot has been described in the previous section. The results shown in Fig. 6b correspond to projection of surface on force $-\gamma$ plane, hence plotted lines refer to constant velocity. We can observe that for small velocities the influence of $\gamma$ parameter is not very significant but with an increase of velocity the influence become noteworthy and strongly nonlinear with hardening character. We assume that the origin of the non-linearities lies in the properties of the fluid inside the dashpot and its flow through a throttling valve of a variable cross-section. A detailed description of the dashpot from the viewpoint of fluid mechanics is beyond the scope of this work. As it is 
easy to see, the matching of data is very good and we claim that the presented model can be used in to simulate the behavior of the dashpot in real life implementations.

\section{Influence of the stepper motor control}

In the investigated dashpot the control of damping coefficient is obtained through the usage of stepper motor that changes the position of the throttling valve. Thus, the changes are not continuous which may affect the stability of operation and induce plethora of nonlinear effect. Still, the model proposed in Sect. 4 does not include this important factor. To investigate if the model can still reproduce the behavior of the real device we perform dedicated experimental test. We fix the velocity of the piston rod the the damper to $0.13\left(\frac{\mathrm{m}}{\mathrm{s}}\right)$, which is an equivalent of $400(\mathrm{rpm})$ on the servomotor. For selected velocity, the piston rod is moving with the assumed constant velocity for $0.22(\mathrm{~s})$ (the test has the same course as presented in Fig. 2 in Sect. 3.2). Based on our tests, the change of full range of damping coefficient (from $\gamma=0(-)$ to $\gamma=130(-))$ takes $0.34(\mathrm{~s})$, so in order to perform the assumed test, we have to vary the control parameter $\gamma$ in two periods of constant piston rod motion. The experiment starts from accelerating the dashpot piston

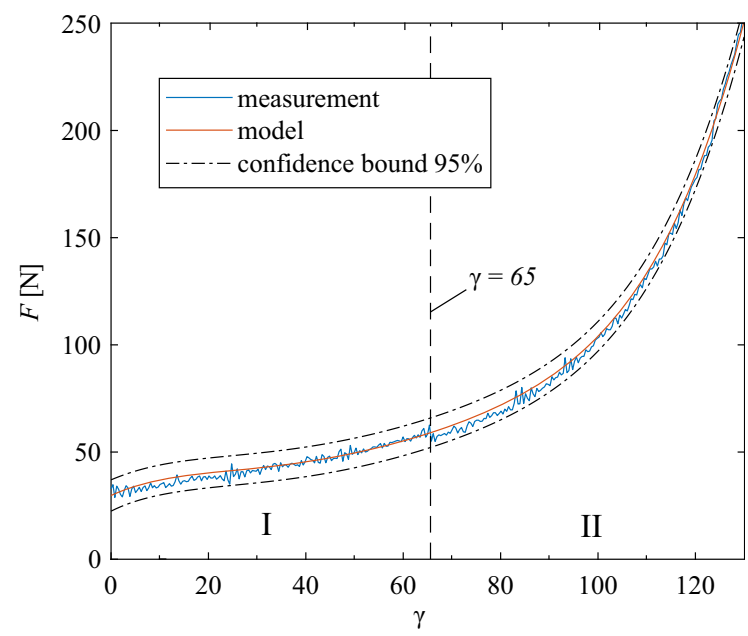

Fig. 7 Force generated by the dashpot as the function of controlling parameter $\gamma$ with constant velocity $v=0.13\left(\frac{\mathrm{m}}{\mathrm{s}}\right)$. Blue and red lines show experimental and numerical results respectively. Dash-dot lines show the $95 \%$ confidence bound calculated for numerical data rod to the constant velocity. After a short stabilization time, we change the dashpot control parameter from $\gamma=0(-)$ to $\gamma=65(-)$, then decelerate the dashpot rod to the full stop. After that, we reverse motion, go back to the starting position and accelerate the rod once again to the same constant velocity, followed by the second change of the dashpot control parameter from $\gamma=65(-)$ to $\gamma=130(-)$. Finally, we decelerate the rod to the full stop. The change of the dashpot control parameter is logged on the data acquisition system in parallel with the force. By combining two curves with respect to the variable dashpot control parameter $\gamma$, we obtained results presented in Fig. 7 . We show the comparison of the experimental result (blue continuous line) and the response calculated based on mathematical model (red continuous line). On both sides of the numerical trace of the force we the plot dash-dot lines which show the $95 \%$ confidence bound for future observations. The experimental results do not cross any confidence bound lines. Hence, we claim that the matching of experimental and numerical data is very good. Thus, we effects of the stepper motor control are minor and the investigated dashpot can be modeled as we propose in Sect. 4 also for applications with continuous change of controlling parameter $\gamma$.

Another important factor is the possible rate of change of the damping coefficient. In the considered device the change of control parameter $\gamma$ from its minimum to maximum value takes at least 0.34 (s). In our test we executed the change from minimum to maximum value in $0.44(\mathrm{~s})$ which refers to $77 \%$ of maximum rate of change and can be considered as fast change in damping coefficient. Although the stepper motor enables fast changes without affecting the dynamics of the system one has to bear in mind the maximum possible rate of change of damping coefficient.

\section{Conclusions}

In this paper we show the comprehensive experimental investigation of the semi-active dashpot. In order to examine its characteristics we create a specially designed laboratory rig. Using a simple helical spring we legitimize the concept of the rig and prove that it enables high precision measurements to examine 
elementary mechanical components of dynamical systems like springs, dampers and inerters

Then, we preform comprehensive experimental test of the semi-active dashpot with a throttling valve Ohlins SD 043. Based on the obtained experimental data we develop a mathematical model of the device. Its parameters are identified using two dimensional surface fit implemented in Matlab. We present the force-velocity and force-control parameter $\gamma$ dependence graphs combined with experimental data. In both cases we obtain very good matching. We observe that the dashpot characteristic is strongly non-linear of hardening type. Hence, for low velocities and small values of $\gamma$ it is close to linear, but with the increase of those parameters it becomes significantly steeper and clearly diverges from linear characteristic. We also investigate the effects of noncontinuous changes of damping coefficient performed by a stepper motor. The results show that the investigated device is capable of fast changes of damping coefficient and without disturbing smooth operation. This proves the robustness of the device and the proposed mathematical model. According to the good agreement between the experimental and simulation results we claim that our model is accurate enough to be used in simulations of dynamical systems equipped with a controllable dashpot with a throttling valve.

Acknowledgements This work has been supported by National Science Centre, Poland - Project No. 2015/17/B/ST8/ 03325 (P. Perlikowski is PI of project).

\section{Compliance with ethical standards}

Conflict of interest Przemyslaw Perlikowski is Guest Editor of SI "Recent Advances in Nonlinear Dynamics and Vibrations". Others authors declare that they have no conflict of interest.

Open Access This article is licensed under a Creative Commons Attribution 4.0 International License, which permits use, sharing, adaptation, distribution and reproduction in any medium or format, as long as you give appropriate credit to the original author(s) and the source, provide a link to the Creative Commons licence, and indicate if changes were made. The images or other third party material in this article are included in the article's Creative Commons licence, unless indicated otherwise in a credit line to the material. If material is not included in the article's Creative Commons licence and your intended use is not permitted by statutory regulation or exceeds the permitted use, you will need to obtain permission directly from the copyright holder. To view a copy of this licence, visit http://creativecommons.org/licenses/by/4.0/.

\section{References}

1. Nashif AD, Jones DI, Henderson JP (1985) Vibration damping. Wiley, New York

2. Makris N, Roussos Y, Whittaker AS, Kelly JM (1998) Viscous heating of fluid dampers. ii: large-amplitude motions. J Eng Mech 124(11):1217-1223

3. Lee D, Taylor DP (2001) Viscous damper development and future trends. Struct Des Tall Build 10(5):311-320

4. Balkanlou VS, Karimi MRB, Azar BB, Behravesh A (2013) Evaluating effects of viscous dampers on optimizing seismic behavior of structures. Int $\mathbf{J}$ Curr Eng Technol 3(4):1150-1157

5. Bergeot B, Bellizzi S, Cochelin B (2017) Passive suppression of helicopter ground resonance using nonlinear energy sinks attached on the helicopter blades. J Sound Vib 392:41-55

6. Rittweger A, Albus J, Hornung E, Öry H, Mourey P (2002) Passive damping devices for aerospace structures. Acta Astronaut 50(10):597-608

7. Saleh M, Sedaghati R, Bhat R (2017) Crashworthiness study of helicopter skid landing gear system equipped with a magnetorheological energy absorber. In: ASME 2017 conference on smart materials, adaptive structures and intelligent systems, American Society of Mechanical Engineers Digital Collection

8. Ahmed M, Yusoff A, Romlay F (2019) Adjustable valve semi-active suspension system for passenger car. Int $\mathbf{J}$ Automot Mech Eng 16(2):6470-6481

9. Zuo L, Zhang P-S (2013) Energy harvesting, ride comfort, and road handling of regenerative vehicle suspensions. J Vib Acoust 135(1):011002-1-011002-8

10. Rodrigues FA, Thouverez F, Gibert C, Jezequel L (2003) Chebyshev polynomials fits for efficient analysis of finite length squeeze film damped rotors. J Eng Gas Turbines Power 125(1):175-183

11. Turnip A, Hong K-S, Park S (2008) Control of a semi-active mr-damper suspension system: a new polynomial model. IFAC Proc 41(2):4683-4688

12. Wei M, Lin K, Guo Q (2018) Modeling mechanical properties of a shear thickening fluid damper based on phase transition theory. EPL (Europhys Lett) 121(5):50001

13. Choi S-B, Nam M-H, Lee B-K (2000) Vibration control of a $\mathrm{mr}$ seat damper for commercial vehicles. J Intell Mater Syst Struct 11(12):936-944

14. Yao G, Yap F, Chen G, Li W, Yeo S (2002) Mr damper and its application for semi-active control of vehicle suspension system. Mechatronics 12(7):963-973

15. Maciejewski I, Krzyzynski T, Meyer L (2014) Control system synthesis of seat suspensions used for protection of working machine operators. Veh Syst Dyn 52(11):1355-1371

16. Dyke S, Spencer B Jr, Sain M, Carlson J (1998) An experimental study of $\mathrm{mr}$ dampers for seismic protection. Smart Mater Struct 7(5):693

17. Occhiuzzi A, Spizzuoco M, Serino G (2003) Experimental analysis of magnetorheological dampers for structural control. Smart Mater Struct 12(5):703 
18. Yanik A, Aldemir U (2019) A simple structural control model for earthquake excited structures. Eng Struct 182:79-88

19. Wang L, Liang Z, Cai M, Zhang Y, Yan J (2019) Adaptive structural control of floating wind turbine with application of mr damper. Energy Procedia 158:254-259

20. Park S, Lackner MA, Pourazarm P, Rodríguez Tsouroukdissian A, Cross-Whiter J (2019) An investigation on the impacts of passive and semiactive structural control on a fixed bottom and a floating offshore wind turbine. Wind Energy 22(11):1451-1471

21. Li D, Botto D, Xu C, Liu T, Gola M (2019) A micro-slip friction modeling approach and its application in underplatform damper kinematics. Int J Mech Sci 161:105029

22. Umer M, Botto D (2019) Measurement of contact parameters on under-platform dampers coupled with blade dynamics. Int J Mech Sci 159:450-458

23. Spelta C, Previdi F, Savaresi SM, Fraternale G, Gaudiano N (2009) Control of magnetorheological dampers for vibration reduction in a washing machine. Mechatronics 19(3):410-421

24. Sajedipour D, Behbahani S, Tabatabaei SMK (2010) Mechatronic modeling and control of a lathe machine equipped with a MR damper for chatter suppression. In: IEEE ICCA 2010. IEEE, pp 802-807

25. Kishore R, Choudhury SK, Orra K (2018) On-line control of machine tool vibration in turning operation using electromagneto rheological damper. J Manuf Process 31:187-198

26. Facey WB, Rosenfeld NC, Choi Y-T, Wereley NM, Choi SB, Chen P (2005) Design and testing of a compact magnetorheological damper for high impulsive loads. In: Electrorheological fluids and magnetorheological suspensions (Ermr 2004). World Scientific, pp 638-644

27. Saleh M, Sedaghati R, Bhat R (2019) Design optimization of a bi-fold mr energy absorber subjected to impact loading for skid landing gear applications. Smart Mater Struct 28(3):035031

28. Graczykowski C, Faraj R (2019) Development of control systems for fluid-based adaptive impact absorbers. Mech Syst Signal Process 122:622-641
29. Choi S-B, Hong S-R, Sung K-G, Sohn J-W (2008) Optimal control of structural vibrations using a mixed-mode magnetorheological fluid mount. Int J Mech Sci 50(3):559-568

30. Kim J-H, Oh J-H (2001) Development of an above knee prosthesis using mr damper and leg simulator. In: Proceedings 2001 ICRA. IEEE international conference on robotics and automation (Cat. No. 01CH37164), vol 4. IEEE, pp 3686-3691

31. Xie H-L, Liang Z-Z, Li F, Guo L-X (2010) The knee joint design and control of above-knee intelligent bionic leg based on magneto-rheological damper. Int J Autom Comput 7(3):277-282

32. Guan X, Ru Y, Huang Y (2019) A novel velocity selfsensing magnetorheological damper: design, fabricate, and experimental analysis. J Intell Mater Syst Struct 30(4):497-505

33. Wilson NL, Wereley NM, Hu W, Hiemenz GJ (2013) Analysis of a magnetorheological damper incorporating temperature dependence. Int J Veh Des 63(2-3):137-158

34. Mohankumar D, Sabarish R, PremJeyaKumar M (2018) Variable damping force shock absorber. Int J Pure Appl Math 118(18):945-955

35. Gong M, Chen H (2019) Variable damping control strategy of a semi-active suspension based on the actuator motion state. J Low Freq Noise Vib Active Control. https://doi.org/ 10.1177/1461348418825416

36. Brzeski P, Lazarek M, Perlikowski P (2017) Experimental study of the novel tuned mass damper with inerter which enables changes of inertance. J Sound Vib 404:47-57

37. Lazarek M, Brzeski P, Perlikowski P (2018) Design and identification of parameters of tuned mass damper with inerter which enables changes of inertance. Mech Mach Theory 119:161-173

38. Lazarek M, Brzeski P, Perlikowski P (2019) Design and modeling of the cvt for adjustable inerter. J Frankl Inst 356(14):7611-7625

Publisher's Note Springer Nature remains neutral with regard to jurisdictional claims in published maps and institutional affiliations. 